\section{Estratégia Saúde da Família e a atenção ao idoso: experiências em três municípios brasileiros}

\author{
The Family Health Strategy and healthcare for the \\ elderly: experiences in three Brazilian cities
}

\section{Abstract}

This study focuses on healthcare for the elderly under the Family Health Strategy in three municipalities (counties) in the State of Rio de Janeiro, Brazil, specifically analyzing the organization of the healthcare network and the knowledge of six health teams, using interviews and focus groups. The study emphasized the central role of work by community health agents, the prioritization of program activities as compared to management of problems in the community covered by the strategy, a work overload in the health teams, disorganization of the healthcare networks, and lack of systemic approaches based on the basic contents of Geriatrics and Gerontology. The article concludes that there is a need for a healthcare network for the elderly consisting of hierarchically organized services and levels of care that provide support for the healthcare teams' work. The teams could also benefit from continuing education programs. The undergraduate medical curriculum should also include more course content in geriatrics and gerontology.

Old Age Assistance; Health of the Elderly; Family Health; Human Resource Formation
Luciana Branco da Motta 1,2

Adriana Cavalcanti de Aguiar 1,3

Célia Pereira Caldas 1

\section{Introdução}

O envelhecimento populacional brasileiro é um desafio para o Sistema Único de Saúde (SUS). A associação entre envelhecimento populacional e o alto custo do cuidado às doenças crônicas vem sendo estudada nacional e internacionalmente 1. Porém, além dos determinantes econômicos do cuidado à saúde do idoso, é importante investigar a natureza e qualidade do cuidado prestado. Uma assistência baseada no consumo intensivo de tecnologia no ambiente hospitalar enfraquece a prevenção e o acompanhamento duradouro a problemas crônicos, permeados por agravantes de natureza psicossocial.

Coloca-se também a questão de quem e como cuidar dos idosos brasileiros. Nos currículos dos cursos de graduação em saúde é pequena a expressão da Gerontologia e Geriatria. O escasso valor social conferido aos idosos na sociedade parece minimizar o apelo da especialidade para estudantes que priorizam o status profissional. Além disso, a literatura especializada é unânime em afirmar que geriatras e/ou gerontólogos não devem ser responsáveis por toda a atenção ao idoso, necessitando trabalhar em conjunto com outros profissionais em diversos níveis de atenção 2 .

No Brasil, a Estratégia Saúde da Família (ESF) foi planejada para reorientar a atenção à saúde da população, fomentando a qualidade de vida, por exemplo, mediante a promoção do envelhe- 
cimento saudável. Como o envelhecimento não é um processo homogêneo, necessidades e demandas dos idosos variam, sendo preciso fortalecer o trabalho em rede para contemplar a atenção aos idosos saudáveis e atender àqueles com diferentes graus de incapacidade ou enfermidade, inclusive nos domicílios. Assim, o adequado cuidado ao idoso demanda um sistema de saúde coordenado, com cada instância contribuindo para as ações das demais.

A reorientação do modelo assistencial mediada pela ESF implica a coordenação com níveis de atenção secundário e terciário, além da expansão e fortalecimento da oferta de atenção básica. No seu atual contexto de implantação, todavia, a rede de saúde de municípios e estados ainda enfrenta inúmeros obstáculos para oferecer o apoio necessário àqueles que atuam na ESF.

Com dezenas de milhares de equipes implantadas - 29,3 mil equipes/2008 - (Ministério da Saúde; http://dab.saude.gov.br/abnumeros / historico_2008.pdf, acessado em 02/Jul/2010), profissionais da ESF enfrentam desafios inerentes ao cuidado à saúde de um crescente número de idosos e sua problemática específica, competindo por atenção com crianças, gestantes, homens e mulheres em idade fértil, num contexto epidemiológico de coexistência de doenças crônico-degenerativas, infecciosas e agravos decorrentes de problemas sociais, especialmente a violência. Por isso, variáveis como apoio familiar e redes de suporte social também devem ser consideradas na análise das condições de vida e do risco de fragilização da população idosa, numa abordagem intersetorial capaz de identificar e atender as necessidades desta população, que em 2009 se aproximava dos 19 milhões de brasileiros (Instituto Brasileiro de Geografia e Estatística; http://www.ibge.gov.br/series_esta tisticas / tema.php?idtema=6, acessado em 02 / Jul/2010).

Esta pesquisa, realizada em três municípios do Estado do Rio de Janeiro, analisou a atenção ao idoso no trabalho da ESF. Com base na descrição das características do processo de trabalho, investigou o conhecimento e a percepção dos profissionais das equipes, e problematizou as dificuldades apontadas na atuação junto aos idosos, discutindo o papel da ESF na rede de atenção ao idoso no Brasil hoje.

\section{Metodologia}

O presente estudo, qualitativo e exploratório, foi aprovado pela Comissão de Ética da Universidade do Estado do Rio de Janeiro. A coleta de dados sobre processo de trabalho baseou-se na construção de fluxograma descritor ${ }^{3}$ e na discussão de situação-problema com as equipes estudadas, empregando a técnica de grupos focais. Percepções dos profissionais foram obtidas por meio de entrevistas individuais com pessoas atuantes em equipes de saúde da família nos três municípios, cuja seleção priorizou o critério de heterogeneidade: incluiu um município de pequeno porte (Engenheiro Paulo de Frontin), um de porte médio (Itaboraí) e um de grande porte (Rio de Janeiro).

Em cada município foram selecionadas, junto ao gestor municipal, duas equipes cujos profissionais tivessem trabalhado em conjunto por um ano, no mínimo, num total de seis equipes. As entrevistas individuais seguiram um roteiro semiestruturado e contaram com a participação de todos os membros das seis equipes investigadas, num total de 54 pessoas: 30 agentes comunitários de saúde, 12 profissionais de nível superior e 12 profissionais de nível médio.

A triangulação 4 de entrevistas individuais com os fluxogramas e resultados dos grupos focais para discussão de situação-problema descortinou aspectos das condições concretas de produção de práticas em saúde, além da atribuição de significados por profissionais e equipes. A análise de conteúdo 5 das entrevistas individuais utilizou categorias analíticas oriundas dos discursos, num processo indutivo apoiado pelo programa de gerenciamento de dados textuais "LOGOS" 6.

\section{Resultados}

Por ocasião da coleta de dados, em 2008, o Município de Engenheiro Paulo de Frontin tinha cerca de 13 mil habitantes e $100 \%$ de cobertura pela ESF. O Município de Itaboraí contava com cerca de 230 mil habitantes, sendo $68 \%$ cobertos pela ESF. A capital do estado, Município do Rio de Janeiro, com cerca de 6,2 milhões de habitantes, apresentava apenas $8 \%$ de cobertura. Apesar dessas diferenças, a construção e análise de fluxogramas descrevendo as atividades das equipes nas unidades de saúde e comunidades evidenciou muitas semelhanças entre municípios e entre unidades do mesmo município, destacando-se a centralidade do trabalho dos agentes comunitários de saúde (ACS) para a atenção aos idosos. Sua função inclui cadastrar, acompanhar as famílias e intermediar a relação entre idosos, famílias e equipes. É sua atribuição registrar os idosos que foram hospitalizados e/ou regressaram de internação hospitalar, e identificar aqueles que necessitam de assistência domiciliar, mediante critérios acordados localmente pelas equipes. 
Segundo uma médica entrevistada, "Eu nunca pensei que um ACS fosse tão importante. (...). São eles que têm o mapa da mina e vão te orientando para as necessidades dos usuários".

Nos dois municípios maiores, com cobertura parcial, prioridades de acesso são mediadas pelas ações programáticas do Ministério da Saúde. O grande número de adscritos ocasiona uma triagem dos usuários que serão acompanhados pelas ações programáticas e/ou que apresentam doenças mais graves. A sobrecarga de usuários impossibilita maior investimento de tempo das equipes em práticas preventivas, ficando a cargo dos ACS acompanhar os usuários nãocadastrados nos programas, estimando riscos e agendando consultas, no caso de necessidades identificadas.

Todos os municípios pesquisados enfrentam dificuldades de coordenação entre os níveis de atenção. Mecanismos de referência e contrarreferência funcionam precariamente. Raramente informações acerca de usuários encaminhados retornam para a Unidade de Saúde da Família. Segundo uma médica entrevistada, “Os pacientes já perceberam isto. Na ESF os profissionais estão atuando, mas a rede não funciona. Isto tira a credibilidade do trabalho". Na tentativa de resolver os problemas, os entrevistados relataram utilizar contatos pessoais para conseguir exames ou consultas, numa referência informal para outras unidades de saúde. Essa desarticulação da rede e a inexistência ou desconhecimento dos serviços de retaguarda disponíveis, além de comprometer a resolutividade da atenção básica, desgastam os profissionais ao acrescentar às suas múltiplas incumbências a de contornar os problemas do sistema.

\section{O debate da situação-problema e as dificuldades do trabalho em equipe}

As equipes de saúde da família selecionadas concordaram em analisar uma situação-problema desenhada para retratar algumas "camadas de diagnóstico” implicadas na identificação e análise de múltiplas comorbidades comuns em idosos, bem como sua abordagem. Foi apresentado às equipes um caso de um idoso com agravos típicos das faixas etárias avançadas: além da emergência de sinais de demência, o Sr. João sofria de hipertensão arterial, diabetes, apresentava déficits sensoriais e contava com pouco suporte familiar. Essa coleta de dados, em formato de grupos focais, contou com a participação da grande maioria dos componentes das equipes, constituindo momentos de troca que permitiram a observação da organização dos grupos. Em quatro das seis equipes o médico conduziu a discussão, excetuando-se as duas nas quais havia rodízio frequente deste profissional. Nessas, o profissional de enfermagem assumiu a coordenação da discussão. Nas seis equipes ficou evidente a intensa mobilização dos ACS com o debate, porém em algumas equipes observou-se maior integração destes agentes por meio de maior participação ativa na discussão.

De modo geral, as equipes identificaram os principais problemas apresentados na situação e apontaram a importância da família, que, portanto, deveria ser orientada e apoiada. Por outro lado, a gravidade da situação do Sr. João e a necessidade de intervenção urgente não foram adequadamente dimensionadas. Por exemplo, o agravamento do déficit cognitivo e os riscos decorrentes do paciente viver sozinho não foram enfatizados, a necessidade de investigação da síndrome demencial e o estabelecimento de uma proposta terapêutica foram pouco explorados. Não houve a sistematização de um plano de cuidado, nem foram estabelecidas prioridades entre as diversas ações, evidenciando que o diagnóstico de componentes do quadro sindrômico não enseja automaticamente uma abordagem sistêmica. Ademais, não foram mencionadas possíveis ações preventivas nem a necessária integração com os demais níveis de atenção da rede, ou ainda o papel de profissionais externos à equipe básica (por exemplo, o assistente social), no apoio a esse usuário e família.

\section{Percepção dos profissionais da ESF acerca da atenção ao idoso}

Após os grupos focais para coleta de dados com base na situação-problema, foi investigada a percepção dos profissionais sobre o trabalho na ESF, especialmente a atenção aos idosos, valendo-se de entrevistas individuais. A análise temática buscou identificar semelhanças e diferenças entre os municípios, bem como entre profissionais de níveis superior e médio/elementar.

Conforme demonstravam os fluxogramas, são os ACS que têm maior contato com os usuários idosos. Para esse grupo, as distâncias a serem percorridas nas visitas domiciliares e o restrito entendimento da comunidade acerca do trabalho foram destacados "Tem muita gente que não entende nosso tipo de trabalho”. Já para médicos e enfermeiros, destacou-se nos três municípios a falta de suporte da rede de saúde: "É a demanda que é muito grande (...). A porta de entrada nas outras unidades em níveis secundários, este acesso é muito restrito (...). [O idoso] não consegue o acompanhamento lá fora". 
$\mathrm{Na}$ literatura da geriatria e gerontologia $7 \mathrm{a}$ atenção ao idoso demanda conhecimento técnico, competências de contextualização do caso no processo de envelhecimento, capacidade de atuação frente à imprevisibilidade e diversidade de situações, bem como o trabalho em equipe multiprofissional. Tentando apreender sentidos da competência profissional para aqueles que atuam no contexto concreto da ESF, foi questionado aos entrevistados o que estes consideram necessário para trabalhar com o usuário idoso. Cada grupo profissional enfatizou aspectos afeitos ao seu processo de trabalho. Os profissionais de nível superior enfatizaram o "conhecimento", pois a eles cabe, em última instância, garantir a resolutividade sobre as demandas em nível local. Os auxiliares destacaram "atenção e cuidado”, atributos associados tradicionalmente ao trabalho do profissional de enfermagem. Para os ACS emergiu o "carinho", bem como "saber escutar". São eles que convivem mais de perto com os usuários, compartilhando, ao menos em parte, seu universo socioeconômico e cultural: "[Os idosos] são muito carentes, precisam de mais carinho, mais conversa”. Chamou atenção o tema "paciência”, apontada por entrevistados de todas as escolaridades e funções: "Eles se queixam bastante, então tem que ter muita paciência" (ACS), ou ainda "Paciência é principal pra eles" (médico).

Refletindo sobre o processo de trabalho nas unidades básicas de saúde (UBS), a sobrecarga das agendas e multiplicidade de ações desenvolvidas, pode-se associar o tema da paciência a problemas diferentes e muitas vezes complementares: necessidade de tempo e conhecimento sobre como lidar com múltiplas queixas e problemas concomitantes, bem como o desenvolvimento de um processo de trabalho multiprofissional que avance na construção da integralidade da atenção.

Os dados sugerem certo grau de banalização da atenção ao idoso, como se somente a atitude de acolhimento fosse suficiente, secundarizando o conteúdo teórico e o desenvolvimento das habilidades específicas: "Eu acho assim, eles têm uma carência muito grande. Eu observo muito, às vezes o paciente idoso vem mais para consulta para conversar, ele não vem nem assim por causa do remédio" (médico). Além da banalização das necessidades aparece certa infantilização desses usuários: trabalhar com idosos "É bem mais fácil porque eles são tão carentesinhos, é tão mais gostoso" (ACS). Por outro lado, emergem as oportunidades de trocas significativas com os idosos, em função de seu maior reconhecimento do trabalho: "Eu acho que [é bom trabalhar com idosos] porque são mais atenciosos” (ACS).
Alguns entrevistados distinguiram o "paciente geriátrico", isto é, o idoso portador de múltiplas comorbidades, em risco ou com perda de capacidade funcional ou cognitiva, do idoso identificado como "basal”. Essa noção aponta para o conhecimento, mesmo que tácito, da identificação da capacidade funcional, síndromes geriátricas e dos idosos frágeis, como componentes da atenção: "Principalmente a questão da violência ao idoso que a gente vê, e a gente se sente desesperado. E também a questão da psiquiatria ao idoso, também me sinto muito despreparado para lidar. Aquele idoso basal a gente não tem dificuldade não, e sim aquele que tem alguma coisa a mais" (médico; grifos nossos).

Quando perguntados sobre a própria capacidade de lidar com o usuário idoso, todos se percebem preparados. Quando não conseguem resolver algum problema, os ACS e auxiliares buscam o apoio dos profissionais de nível superior, principalmente enfermeiros. Esses, por sua vez, em geral consideram-se preparados, mas destacam obstáculos decorrentes da sobrecarga de trabalho. Não apareceu nas narrativas menção à gerontologia e à existência de conhecimento específico acerca do envelhecimento. A atenção aos idosos pareceu estar diluída no meio das diferentes ações programáticas. "A gente se acostumou a separar hipertenso, diabético, puericultura, atendimento à saúde de mulher e esqueceu o idoso e o adolescente" (enfermeira). Houve menção ao despreparo da rede para lidar com os usuários idosos, podendo gerar iatrogenia: "Eu passo antihipertensivo, a pessoa não leva a minha receita ao cardiologista, que passa mesma medicação com outro nome fantasia. A pessoa chega aqui com a pressão baixíssima porque está tomando a dose dupla" (médico).

As narrativas sobre redes de suporte social sugerem desconhecimento ou ceticismo quanto ao seu papel. Além do tema não emergir espontaneamente, quando perguntados diretamente sobre redes apenas a família apareceu nas narrativas. Alguns auxiliares identificam falta de informação sobre os recursos institucionais existentes na área; médicos e enfermeiros se referem ao restrito acesso aos serviços especializados e de suporte social, bem como à desarticulação do sistema de saúde.

O tema da violência contra o idoso assumiu grande importância nas narrativas de todas as categorias profissionais, nos três municípios. O tipo mais identificado foi a violência intrafamiliar, descrita como dificultadora da atenção e impeditiva para a resolutividade das ações: " $A$ pessoa que fica com [a usuária idosa] dá o remédio de segunda a sexta. Aí chega o sábado, a filha não dá porque ela urina muito e dá trabalho" (ACS). 
A falta de conhecimento dos recursos institucionais existentes para lidar com esses casos compromete a abrangência das ações: "Aí soubemos da delegacia do idoso. Eu não sabia que existia isso" (ACS). A notificação de suspeita de violência, obrigatória pelo Estatuto do Idoso ${ }^{8}$, cria situações percebidas como de risco para a equipe: "Como reclamar daquilo? A gente tinha que preencher um papel, botar o nosso nome, todos na capacitação foram contra... a gente ia correr risco... tem que preencher a ficha, colocar o nome, o telefone e o endereço, o setor que você está, microárea... eu jamais vou fazer isso" (ACS). Situações que poderiam ser descritas como de violência institucional aparecem no cotidiano das equipes: "Idosos que andam mal ou ainda conseguem se mobilizar para ir a hospital público reclamam do tempo de espera. Eles querem ser atendidos com uma certa prioridade, isso existe, existe entre aspas, e aí eu tenho essa dificuldade de pedido de exame, que eu peço e não tem como fazer. Eles não gostam de ir ao posto de saúde porque tem que ficar esperando desde a madrugada" (médico).

\section{Discussão}

Este trabalho iluminou pontos relevantes para o aprimoramento da atenção à saúde do idoso na ESF. O envelhecimento populacional e os idosos ainda não recebem a devida atenção do sistema de saúde, reiterando a desvalorização social da velhice. Essa emerge no discurso dos profissionais entrevistados quando a atenção ao idoso é banalizada (enfatizando que querem "carinho e atenção" sem atentar para problemas subjacentes) ou quando os profissionais se referem aos idosos no diminutivo. É um grande desafio para as políticas públicas destacar a importância social do envelhecimento, o que provavelmente facilitaria a inclusão de conteúdos de interesse na formação dos profissionais de saúde.

Apesar da boa vontade dos entrevistados, a frágil apropriação de conhecimentos da geriatria e gerontologia dificulta a abordagem de questões características da atenção ao idoso, como as síndromes geriátricas. Apesar de identificarem componentes do problema, as equipes não dimensionavam corretamente suas consequências imediatas e mediatas, dentro das especificidades do conhecimento acerca do envelhecimento, resultado também verificado por Castro \& Vargas 9 em seu estudo no Município do Rio de Janeiro.

No processo de trabalho das equipes, os ACS assumem boa parte do acompanhamento de usuários (muitos deles idosos) não incluídos nas ações programáticas preconizadas pelo Minis- tério da Saúde. Esforçando-se para identificar situações de risco, marcam consultas e tomam decisões, a partir das necessidades da comunidade ou necessidades percebidas. Dada a pressão da demanda que incide sobre todos os membros da equipe, e da falta de redes de suporte social, muitas vezes esses ACS são pouco apoiados no exercício profissional, o que é agravado pela incompreensão que identificam sobre a natureza de seu trabalho por parte das comunidades.

Este estudo evidencia que a capacitação de profissionais de saúde na ESF é insuficiente para a atenção à saúde do idoso, sendo necessário investir no desenvolvimento de competências para lidar com o desafio do envelhecimento, abrangendo a prevenção, a reabilitação e a melhor compreensão dos determinantes socioambientais do processo saúde/doença. Esse esforço deve extrapolar a formação especializada. Os movimentos de mudança nos cursos de graduação em saúde, balizados pelas Diretrizes Curriculares Nacionais para Cursos de Graduação 10 e nas políticas nacionais voltadas para o idoso - Política Nacional de Saúde da Pessoa Idosa (PNSPI) 11 e Política Nacional do Idoso 12 - são um bom começo, sendo necessário maior respaldo do aparelho formador. A Educação Permanente 13, voltada para a problematização baseando-se na realidade do processo de trabalho, no contexto local é fundamental para o desenvolvimento das equipes e melhor integração dos serviços em redes.

A inexistência de redes de atenção ao idoso apareceu muitas vezes na pesquisa, instigando a problematizar qual é o papel da ESF: reorientar o sistema de saúde a partir da atenção básica não significa atribuir à ESF toda a responsabilidade e encargos que cabem aos três níveis de atenção. $\mathrm{O}$ investimento no desenvolvimento de novas competências para os profissionais da equipe básica não irá substituir as ações que devem ser desempenhadas no nível do ambulatório de especialidades, dos laboratórios e centros de diagnóstico, e dos hospitais gerais e especializados.

Pesquisando o (escasso) apoio institucional para orientação e informação aos cuidadores de idosos com alta dependência, Caldas 14 destacou a importância da rede de suporte formal e informal ser reforçada, a fim de permitir que as famílias assistam seus idosos. No presente estudo, as redes de apoio social não aparecem nas falas, evidenciando desconhecimento dos entrevistados sobre seu papel potencial na assistência aos idosos das comunidades atendidas. Mesmo na discussão da situação-problema, que apontava a necessidade da família do idoso assumir seu cuidado no curto prazo, somente uma equipe observou a falta de estrutura no município para apoiar aquela família. 
Algumas questões político-institucionais demandam ser discutidas devido à determinação que exercem no processo de trabalho. A baixa permanência dos profissionais, principalmente médicos, é recorrente no país 15. Para esses profissionais, aparece uma depreciação do seu trabalho decorrente da pouca importância atribuída por seus próprios pares e pelos usuários, agravada pela remuneração, considerada baixa para a dedicação exclusiva demandada pela ESF. A rotatividade de profissionais se alimenta da precariedade dos vínculos empregatícios, principalmente para os ACS 16. Durante os grupos focais ficaram claras as consequências da falta de integração: naquelas equipes em que a rotatividade foi maior os ACS interagiam pouco com os demais participantes no estabelecimento de um plano de cuidado.

A operacionalização da ESF como reorientadora do sistema de saúde depende também de mudanças na gestão, para incentivar a produção de informações de boa qualidade, planejamento participativo e a legitimidade de pactuações de objetivos e metas no nível loco-regional. A autogestão demanda tomada de decisões no nível local, com base na realidade encontrada na comunidade e no diálogo com o nível central da gestão municipal, que muitas vezes verticaliza decisões e formata programas. O rol de atividades previstas e de ações programadas para os profissionais das equipes de saúde da família é longo e variado, diferindo da prática assistencial habitual e gerando frustração. Criado com o objetivo de ampliar a abrangência e conteúdo das ações e da resolutividade, o Núcleo de Apoio à Saúde da Família (NASF) 17 compreende equipes compostas por profissionais de saúde de diversas áreas e especialidades, não sendo a saúde do idoso priorizada, o que é evidenciado pela ausência de geriatras ou gerontólogos.

Além disso, a PNSPI 13 incumbe a atenção básica, em especial a ESF, de prover atenção domiciliar às famílias adscritas, assim como o acompanhamento de idosos institucionalizados. Em redes pouco estruturadas, recai sobre as equipes básicas a responsabilidade sobre o cuidado de indivíduos dependentes (idosos ou não). Mas a assistência à saúde no domicílio não se encerra levando a equipe à casa do indivíduo. Implica perceber e interpretar adequadamente o contexto familiar, socioeconômico e cultural, extrapolando as fronteiras do modelo biomédico, e demandando a integração das redes de apoio e o desenvolvimento de tecnologias diferenciadas 18 . Isso é reforçado pelo fato de que a violência, em suas múltiplas facetas, permeia o processo de trabalho das equipes estudadas. Seu enfrentamento, porém, requer informação, trabalho em equipe, articulação do sistema de saúde e intervenções intersetoriais.

\section{Considerações finais}

O desenvolvimento de competências para os profissionais de saúde atuarem na atenção básica é necessário para o enfrentamento do envelhecimento populacional. Os conteúdos de geriatria e gerontologia ainda têm pouco peso na formação do profissional de saúde, tanto na graduação como na pós-graduação. Devido à pressão demográfica e epidemiológica, a Educação Permanente pode fortalecer as ações dos profissionais já inseridos no processo de trabalho, iluminando especificidades da atenção ao idoso e facilitando a integração entre os componentes da equipe de saúde da família.

Para que a ESF tenha uma atuação efetiva, cabe discutir o papel dos ACS, investindo em sua capacitação e integração à equipe, o que implica a formalização de seus contratos e o apoio às suas ações. São esses profissionais que fazem a mediação com a comunidade, cujo trabalho permite a identificação precoce de riscos diversos como violência domiciliar, desenvolvimento de incapacidades, identificação de usuários frágeis, com alta dependência, acamados. O trabalho dos ACS pode se beneficiar da utilização de protocolos de simples aplicação, que possam trazer respostas às necessidades e não só identificação de problemas. O excesso de famílias adscritas em algumas equipes traz a necessidade de triagem, com redução do acesso dos usuários às UBS, deixando com o ACS o acompanhamento dos usuários não cadastrados nos programas instituídos pelo Ministério da Saúde. Isso é um fator dificultador para ações de prevenção e promoção de saúde, para a comunidade em geral, principalmente os adultos e idosos.

A construção de uma rede de atenção à saúde do idoso é o maior desafio, por sua natureza complexa. Demanda a oferta de diversos tipos de serviços, que necessitam de fontes de financiamento, às vezes distintos, que devem ser estruturadas de forma regionalizada, integrada, com o objetivo de garantir a longitudinalidade da atenção. A falta de serviços com objetivos distintos como reabilitação, cuidado de enfermagem, cuidado paliativo, cuidado para portadores de incapacidade física e/ou mental fragmenta e dificulta uma assistência longitudinal e cuidadora. Outro limitante apontado nas entrevistas é a falta de transporte que inviabiliza o atendimento a usuários com locomoção prejudicada. A socialização dos indivíduos idosos integra ações de outras esferas que não a saúde, como a Educação 
e Ação Social. Esse tipo de trabalho, voltado para indivíduos considerados saudáveis, demanda parcerias entre instâncias da saúde e aquelas de outras secretarias.

A presença do usuário idoso em uma rede de atenção requer diálogo entre os diferentes serviços de saúde e níveis assistenciais. Daí a importância da capacitação, estabelecimento de mecanismos de referência e contrarreferência, adaptação de instrumentos de identificação de risco de fragilidade e incapacidade funcional (dentro de cada nível assistencial, com ênfase na atenção básica). Cada um dos serviços e níveis assistenciais deve ter claro, tanto para si como para a comunidade, seu espectro de ação. Assim, ficaria mais definido o papel da ESF na prevenção primária e secundária, educação e promoção de saúde, identificação do idoso de risco de fragilização ou perda funcional, o acompanhamento dos idosos acamados ou incapacitados de se locomoverem. O trabalho de prevenção terciária, buscando minimizar sequelas, pode permear ações da ESF mas demandam a implantação de núcleos de apoio em geriatria e gerontologia, garantindo a retaguarda técnica necessária.

A desorganização da rede, a falta de estruturas de apoio, a falta de integração dos serviços existentes e a insuficiente oferta de serviços especializados (como centros-dia ou hospitais-dia, unidades de média e longa permanência com cuidado específico para idosos comprometidos física ou mentalmente, unidades de cuidados paliativos) e de suporte e apoio às famílias e cuidadores ainda incapacitam o SUS para prestar uma atenção integral à saúde do idoso. O contexto atual, no entanto, já apresenta a expansão do acesso a serviços de saúde, cabendo agora debater a importância social dada ao idoso no Brasil, gerando vontade política para desenvolver os necessários equipamentos no sistema, de forma a permitir um trabalho resolutivo e gratificante de profissionais capacitados para tal.

\section{Resumo}

O processo de trabalho, a organização da rede as sistencial e o conhecimento dos profissionais de seis equipes da Estratégia Saúde da Família sobre a atenção ao idoso foram investigados em três municípios do Estado do Rio de Janeiro, Brasil, por meio de entrevistas e de grupos focais. Identificou-se a centralidade do trabalho dos agentes comunitários, a priorização das ações programáticas em detrimento da abordagem dos problemas da comunidade adscrita, a sobrecarga de trabalho, a desorganização das redes de saúde e a falta de abordagens sistêmicas fundamentada em conteúdos básicos da Geriatria e Gerontologia. Conclui-se que existe a necessidade de uma rede de atenção ao idoso composta por serviços e níveis de atenção hierarquizados que ofereça suporte às ações das equipes. Essas, também podem se beneficiar de programas de educação permanente, sendo necessário, além disto, aumentar a oferta de conteúdos de geriatria e gerontologia nos cursos de graduação.

Assistência a Idosos; Saúde do Idoso; Saúde da Família; Formação de Recursos Humanos

\section{Colaboradores}

L. B. Motta contribuiu com a concepção do estudo, projeto, coleta, análise e interpretação dos dados e redação do artigo. A. C. Aguiar contribuiu com a concepção do estudo, projeto, análise e interpretação dos dados e redação do artigo. C. P. Caldas contribuiu com a concepção do estudo, análise, discussão dos dados e redação do artigo. 


\section{Referências}

1. Boult C, Boult L, Pacala JP. Systems of care for older populations of the future. J Am Geriatr Soc 1998; 46:499-505.

2. Hazzard WR, Woolard N, Regenstreif DI. Internal medicine: at the nexus of the health care system in responding to the demographic imperative of an aging population. Am J Med 2001; 110:507-13.

3. Franco TB. Fluxograma descritor e projetos terapêuticos para análise de serviços de saúde, em apoio ao planejamento: o caso de Luz (MG). In: Merhy EE, Magalhães Júnior HM, Rimoli J, Franco TB, Bueno WS, organizadores. O trabalho em saúde: olhando e experimentando o SUS no cotidiano. 4ạ Ed. São Paulo: Editora Hucitec; 2007. p. 161-98.

4. Minayo MCS, Assis SG, Souza ER. Avaliação por triangulação de métodos: abordagem de programas sociais. Rio de Janeiro: Editora Fiocruz; 2005.

5. Bardin L. Análise de conteúdo. Lisboa: Edições 70; 1977.

6. Camargo Júnior KR. Apresentando Logos: um gerenciador de dados textuais. Cad Saúde Pública 2000; 16:286-7.

7. Motta LB, Aguiar AC. Novas competências profissionais em saúde e o envelhecimento populacional brasileiro: integralidade, interdisciplinaridade e intersetorialidade. Ciênc Saúde Coletiva 2007; 12:363-72.

8. Senado Federal. Parecer $n^{\circ} .1301$ de 2003. Redação final do Projeto de Lei da Câmara no. 57, de 2003 (no. 3.561, de 1997, na Casa de origem). Brasília: Comissão Diretora, Senado Federal; 2003.

9. Castro MR, Vargas LA. A interação/atuação da equipe do Programa de Saúde da Família do Canal do Anil com a população idosa adscrita. Physis (Rio J.) 2005; 15:329-51.

10. Conselho Nacional de Educação. Resolução CNE/ CES no. 4, de 7 de novembro de 2001. Institui as Diretrizes Curriculares Nacionais do curso de graduação em Medicina. Brasília: Câmara de Educação Superior, Conselho Nacional de Educação; 2001.
11. Ministério da Saúde. Portaria no. 2.528, de 2006. Aprova Política Nacional da Saúde da Pessoa Idosa. Diário Oficial da República Federativa do Brasil 2006; 20 out.

12. Ministério da Justiça. Lei no. 8.842. Dispõe sobre a Política Nacional do Idoso, cria Conselho Nacional do Idoso e dá outras providências. Diário Oficial da República Federativa do Brasil 1994; 6 jan.

13. DeLuiz N. Qualificação, competências e certificação: visão do mundo do trabalho. Formação 2001; (2):5-15.

14. Caldas CP. O idoso em processo de demência: o impacto na família. In: Minayo MCS, Coimbra Jr. CEA, organizadores. Antropologia, saúde e envelhecimento. Rio de Janeiro: Editora Fiocruz; 2002. p. 51-71.

15. Guglielmi MC. A política pública "Saúde da Família" e a permanência fixação do profissional em Medicina: um estudo de campo em Pernambuco [Tese de Doutorado]. Rio de Janeiro: Instituto de Medicina Social, Universidade do Estado do Rio de Janeiro; 2006.

16. Pedrosa JIS, Teles JBM. Consenso e diferenças entre equipes do Programa Saúde da Família. Rev Saúde Pública 2001; 35:303-11.

17. Ministério da Saúde. Portaria no. 154, de 24 de janeiro de 2008. Cria NASF - Núcleos de Apoio à Saúde da Família. Diário Oficial da República Federativa do Brasil 2008; 25 jan.

18. Giacomozzi CM, Lacerda MR. A prática de assistência domiciliar dos profissionais da estratégia de saúde da família. Texto \& Contexto Enferm 2006; 15:645-53.

Recebido em 24/Jul/2010

Aprovado em 15/Fev/2011 